\title{
Effect of Heart Rate on CT Angiography Using the Enhanced Cardiac Model of the 4D NCAT
}

\author{
W.P. Segars ${ }^{*}{ }^{a}$, K.Taguchi ${ }^{a}$, G.S.K. Fung ${ }^{\mathrm{a}, \mathrm{b}}$, E.K. Fishman ${ }^{\mathrm{a}}$, and B.M.W. Tsui ${ }^{\mathrm{a}}$ \\ ${ }^{a}$ The Russell H. Morgan Department of Radiology and Radiological Science, Johns Hopkins \\ University, Baltimore, MD; ${ }^{b}$ Department of Electrical and Electronic Engineering, University of \\ Hong Kong, Pokfulam Rd, Hong Kong
}

\begin{abstract}
We investigate the effect of heart rate on the quality and artifact generation in coronary artery images obtained using multi-slice computed tomography (MSCT) with the purpose of finding the optimal time resolution for data acquisition. To perform the study, we used the 4D NCAT phantom, a computer model of the normal human anatomy and cardiac and respiratory motions developed in our laboratory. Although capable of being far more realistic, the 4D NCAT cardiac model was originally designed for low-resolution imaging research, and lacked the anatomical detail to be applicable to high-resolution CT. In this work, we updated the cardiac model to include a more detailed anatomy and physiology based on high-resolution clinical gated MSCT data. To demonstrate its utility in high-resolution dynamic CT imaging research, the enhanced 4D NCAT was then used in a pilot simulation study to investigate the effect of heart rate on CT angiography. The 4D NCAT was used to simulate patients with different heart rates (60-120 beats/minute) and with various cardiac plaques of known size and location within the coronary arteries. For each simulated patient, MSCT projection data was generated with data acquisition windows ranging from 100 to $250 \mathrm{~ms}$ centered within the quiet phase (mid-diastole) of the heart using an analytical CT projection algorithm. CT images were reconstructed from the projection data, and the contrast of the plaques was then measured to assess the effect of heart rate and to determine the optimal time resolution required for each case. The 4D NCAT phantom with its realistic model for the cardiac motion was found to provide a valuable tool from which to optimize CT cardiac applications. Our results indicate the importance of optimizing the time resolution with regard to heart rate and plaque location for improved CT images at a reduced patient dose.
\end{abstract}

Keywords: CT, Phantom, Simulation

\section{INTRODUCTION}

Helical multi-slice $\mathrm{CT}$ is the latest advance in $\mathrm{x}$-ray $\mathrm{CT}$ imaging [1]. MSCT allows for rapid cardiac imaging during a single breath-hold offering a new, exciting approach from which to evaluate cardiovascular disease. With this ability, MSCT has been utilized for the detection or quantification of calcified coronary plaques, CT angiography [2] of the coronary arteries, and 4D functional cardiac imaging. Despite the impressive performance of current MSCT scanners, however, the temporal resolution of individual images (50 to $250 \mathrm{~ms}$ depending on the scan and reconstruction protocols) still remains insufficient for eliminating motion artifacts caused by the rapidly moving heart and pulmonary vessels [3].

In this study, we investigate the effect of heart rate on the quality and artifact generation in coronary artery images obtained using helical MSCT with the purpose of finding the optimal time resolution required for different cases. The work is performed through a simulation study using the four-dimensional (4D) NURBS-based cardiac-torso (NCAT) phantom [4-6], a computer model of the human anatomy and cardiac and respiratory motions developed in our laboratory for imaging

Medical Imaging 2006: Physics of Medical Imaging, edited by Michael J. Flynn, Jiang Hsieh, Proceedings of SPIE Vol. 6142, 61420I, (2006) $\cdot 1605-7422 / 06 / \$ 15 \cdot$ doi: $10.1117 / 12.653347$ 

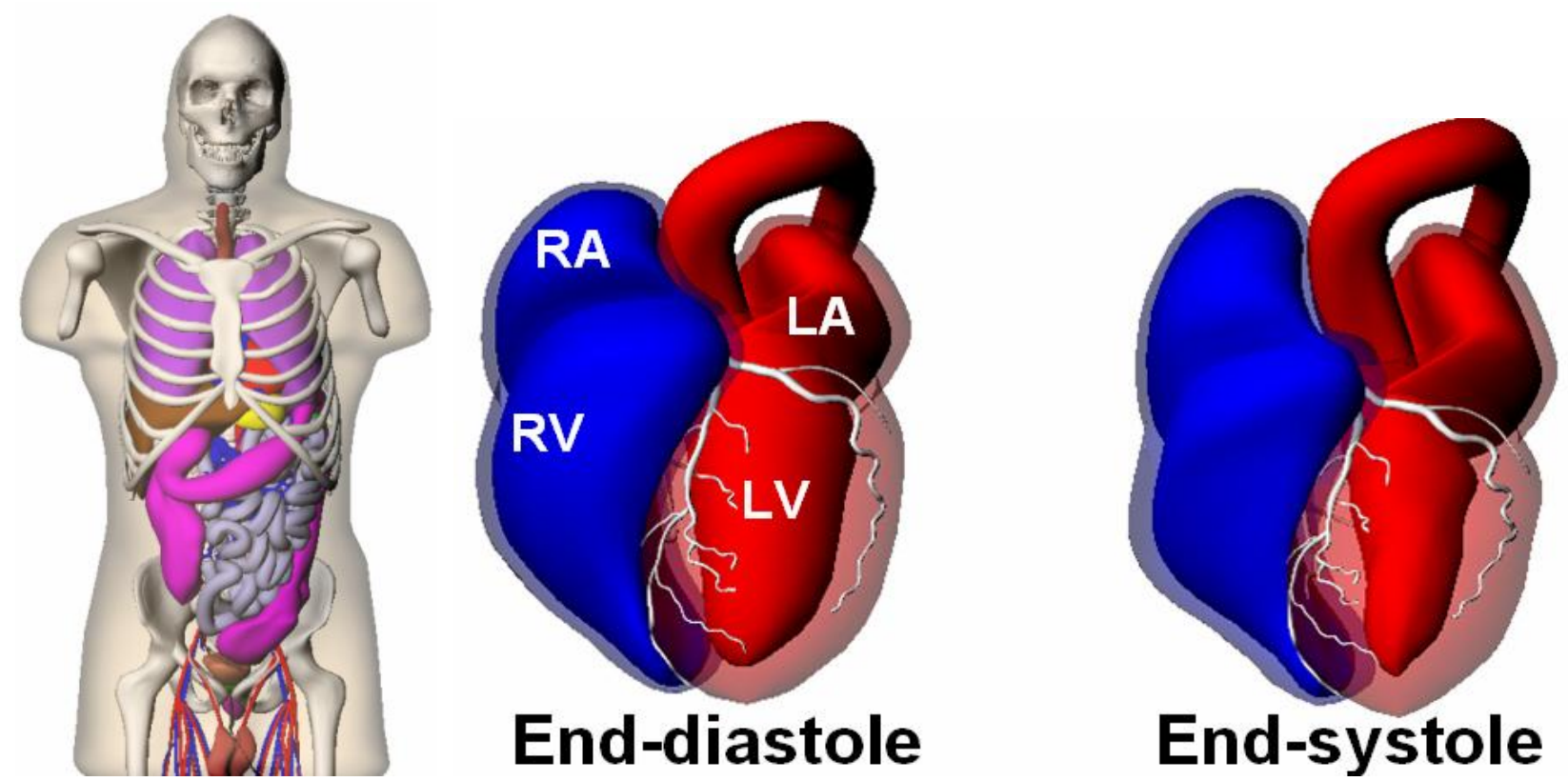

Figure 1: (Left) Anterior view of the 4D NCAT phantom (male version). (Right) Current beating heart model of the 4D NCAT. The heart includes a generic model for the coronary artery tree.

research (Fig. 1). The 4D NCAT was originally designed for low-resolution nuclear medicine imaging research, but is currently being updated to include more anatomical detail to be applicable to high-resolution CT. In this work, the cardiac model of the 4D NCAT is greatly enhanced to include a level of detail suitable for our purposes.

\section{METHODS}

\subsection{Enhancing the 4D NCAT Cardiac Model}

High-resolution gated cardiac MSCT data obtained from a healthy male subject (Fig. 2) was used to enhance the cardiac model of the current 4D NCAT phantom. The data was obtained from Dr. Elliot Fishman of the Johns Hopkins University in accordance with the HIPPA standards. The study consisted of ten time frames over a complete cardiac cycle. At each time frame, the $512 \times 512$ transaxial images had a pixel size of $0.39 \mathrm{~mm} \times 0.39 \mathrm{~mm}$ and a slice thickness of $0.6 \mathrm{~mm}$.

For each time frame, 3D NURBS surfaces were created to model the four chambers of the heart: the right and left ventricles and atria. The heart chambers were manually segmented using the software application SURFdriver [7] to display the time-frame

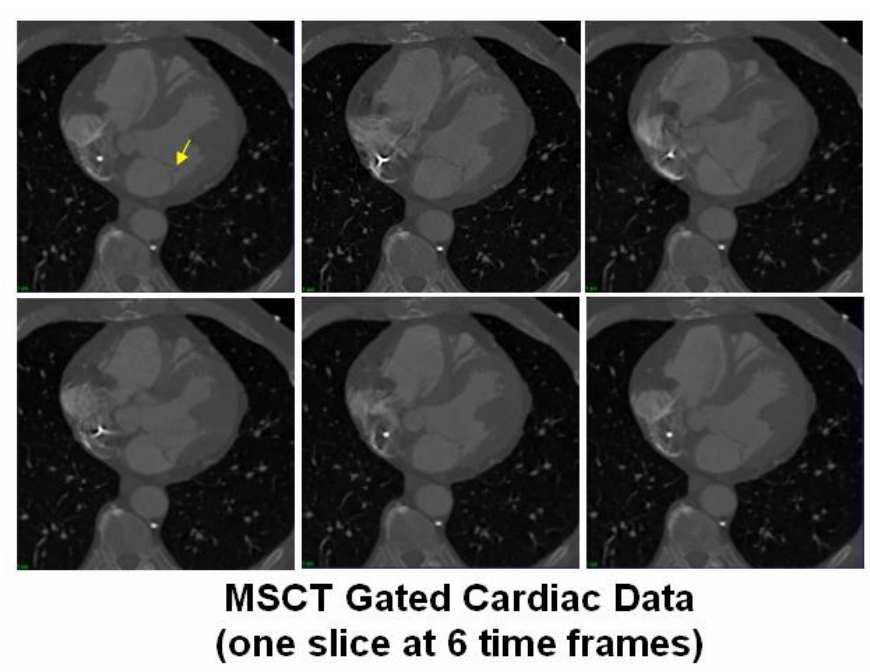

Figure 2: High-resolution gated MSCT cardiac data. The data includes such anatomical details as the coronary vessels and valves of the heart (arrow), and pulmonary vessels. 
transaxial images and define contours for the different structures. 3D NURBS surfaces were then fit to the contours defining each heart structure at each time frame using the Rhinoceros 3D NURBS modeling software [8]. The time correspondence between control points defining a surface over the time frames was set up using the original 4D NCAT cardiac model based on tagged Magnetic Resonance Imaging (MRI) data. This model accurately represents the contracting, twisting motion of the heart that has been previously described using tagged MRI analysis [9-13]. The twisting motion of the heart cannot be ascertained from CT imaging data; therefore, the cardiac twist illustrated in the 4D NCAT was scaled to fit the new heart segmented from the CT data. Once the twisting motion was established, the radial and longitudinal contractions of the control points could be obtained by noting the epi- and endocardial borders in the gated MSCT images. Using this technique, the $3 \mathrm{D}$ position of each control point defining a cardiac surface was determined for each time frame. Time curves were then defined for each control point's position creating a time changing 3D surface or 4D NURBS model for each heart chamber.

In addition to the cardiac chambers, the coronary artery tree, heart valves, and other small details of the heart were modeled. Initial 3D NURBS surface models were created by segmenting the initial time frame of the gated MSCT data. The motion of the different structures throughout the cardiac cycle was determined by tracking landmark points located on or within them for each subsequent time frame. For example, to determine the motion of the coronary vessels, we tracked the branching points at which the vessels divide. From the motion analysis, we created dynamic NURBS models for the heart structures using the 4D NURBS technique previously used to create the initial cardiac and respiratory models of the 4D NCAT phantom [4-6]. Based on the motion of the landmark points, time curves were derived for the $3 \mathrm{D}$ position of the control points that define the different structures creating a 4D NURBS model. The 4D NURBS surfaces for the coronary artery tree and heart valves were then incorporated with those created for the cardiac chambers to create a more realistic and detailed cardiac model for the 4D NCAT phantom.

\subsection{Simulation Study: Effect of Heart Rate on CT Angiography}

The enhanced 4D NCAT was then used to simulate patients with different heart rates (60-120 beats/minute) and with various cardiac plaques of known size and location within the coronary artery tree. Cardiac plaques were modeled as 4D NURBS surfaces lying on the walls of the coronary vessels with the attenuation value set to correspond to that of calcified plaques (average CT number $=1100$ ). The plaques were set to move with the coronary artery tree as the heart beats. Three locations were simulated: proximal right coronary artery (RCA), proximal left anterior descending artery (LAD), and distal LAD. MSCT projection data were generated from each simulated patient using a unique analytic projection algorithm that accurately models the CT imaging process $[6,14]$. Given parameters describing the MSCT scanner (geometry, field of view (FOV), x-ray energy spectrum, number of detector elements and rows, dose, etc.), the projector calculates the CT projection data directly from the NURBS surface definition of the phantom without voxelizing it. Using this algorithm, noise-free MSCT projection data was generated from each phantom using a cone-beam geometry with a fan angle of 48 degrees and a standard chest $\mathrm{x}$ ray energy spectrum with a tube voltage of $120 \mathrm{kVp}$ and $5 \mathrm{~mm}$ aluminum filtration obtained from the Catalogue of Spectral Data for Diagnostic X-rays [15]. Imaging was performed over data acquisition windows ranging from 100 to $250 \mathrm{~ms}$ over a half scan range (thus, a temporal resolution of 80 to $200 \mathrm{~ms} /$ revolution). For each case, the data were acquired over an optimal window centered within the quiet phase (mid-diastole) of the heart. During this brief period, the heart motion is 
reduced. However, the use of longer data acquisition windows (approaching $250 \mathrm{~ms}$ ) includes more and more cardiac motion depending on the duration of the quiet phase. The projection data for each simulated patient was reconstructed into CT images using an ECG-gated halfscan, Feldkamp algorithm. Regions of interest (ROI) were defined for each cardiac plaque and the background in the resulting images. The contrast of the plaques (Contrast plaque $^{-}$ Contrast $_{\text {background }}$ ) as measured from the ROI's were used to assess the effect of heart rate and to determine the optimal time resolution required for each case.

\section{RESULTS}

\subsection{Enhanced Cardiac Model}

Figure 3 shows the enhanced cardiac model of the 4D NCAT phantom defined at end-diastole (ED) and end-systole (ES). The surfaces are shown for each structure of the heart including the four chambers, the cardiac vessels, and the largest branches of the coronary artery tree. Since continuous time curves are defined for each surface, the heart phantom has infinite resolution in the time domain. Any number of time frames can be generated. Using the time curves, thirty time frames were generated from the original ten base frames. The thirty frames illustrate a smoother contracting and twisting motion of the myocardium as compared to the original ten time frames. For each of the thirty frames, the volumes and muscle mass of each of the heart chambers were calculated. The volume curves for the ventricles and atria for the thirty frames are shown in Figure 4 for a heart rate of 60 beats per minute (bpm). The curves follow the normal pattern for the beating heart. The total myocardial mass was found to be conserved within $\pm 5 \%$.
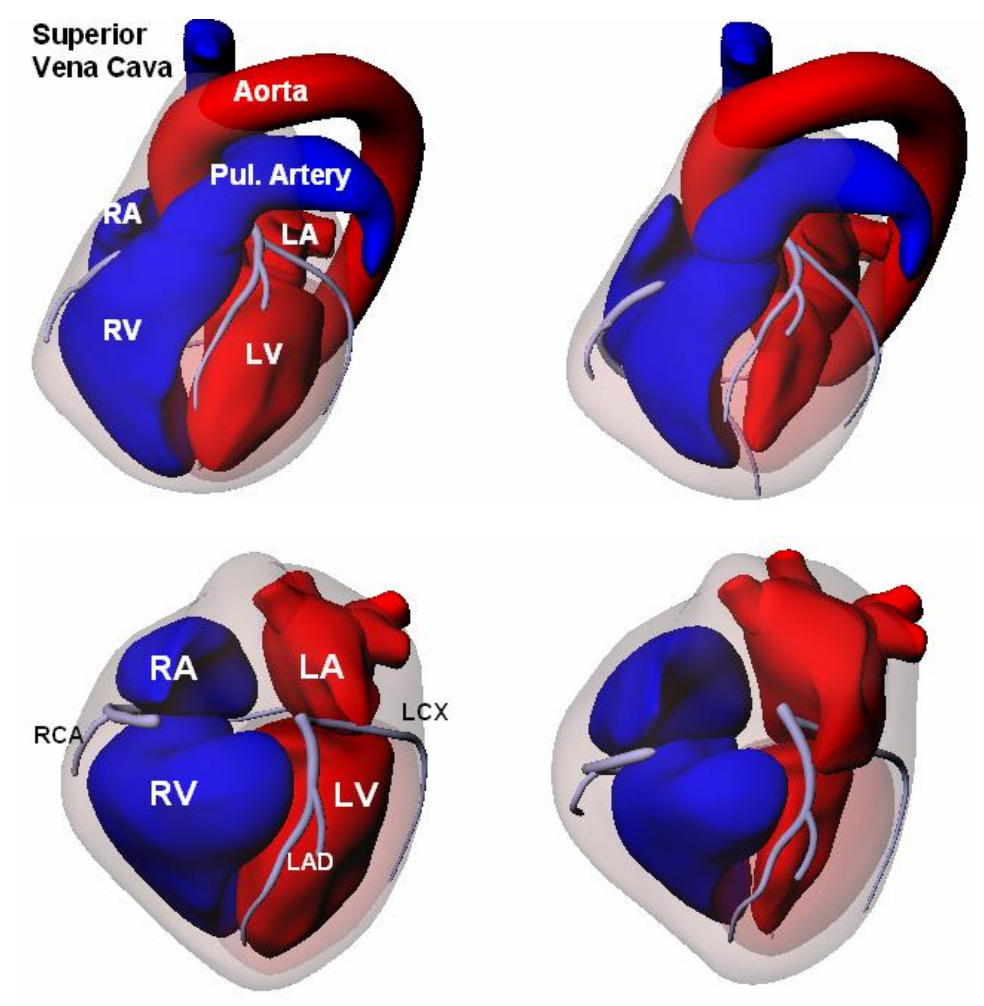

Figure 3: (Top) 3D view of the enhanced model for the beating heart based on the gated MSCT data. (Bottom) Top view of the heart chambers and coronary artery branches. The heart is shown at enddiastole (left) and end-systole (right).

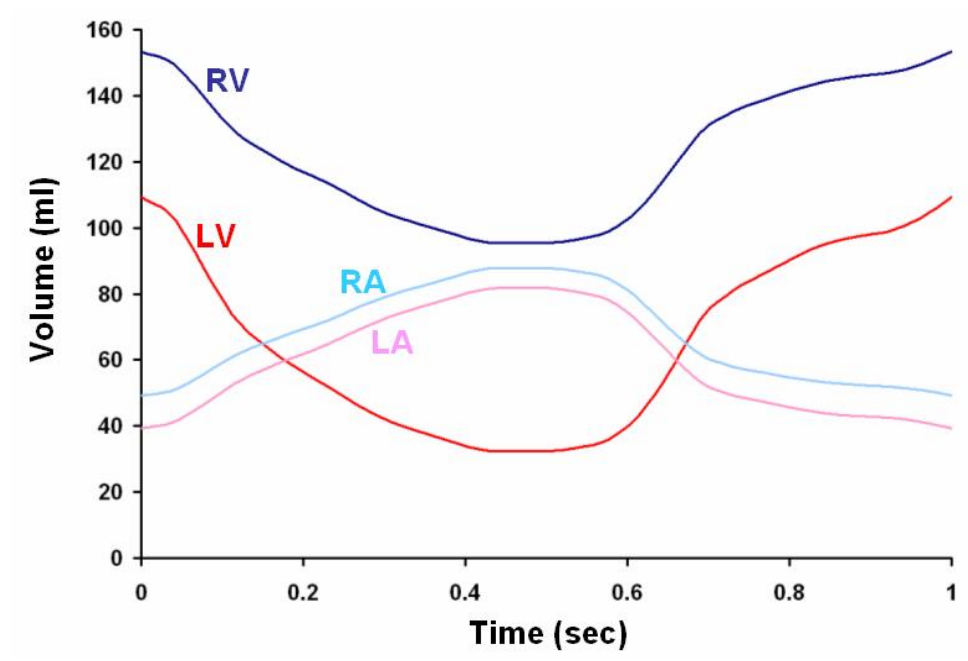

Figure 4: Volume curves from the enhanced 4D NCAT cardiac model. 


\subsection{Simulation Results}

Figure 5 shows the three plaque locations selected for the simulation study. Varying amounts of cardiac motion can be expected at the three locations as shown in Figure 6. The RCA and LCX segments of the arterial tree can be seen to move the most from diastole to systole.

The contrast of the plaques was found to decrease with increasing heart rate and longer data acquisition windows, Figures 7-9. The effect was the most dramatic for the RCA due to the increased motion of this arterial branch during mid-diastole, Figure 10. The contrast of the RCA plaque was found to be reduced by more than $40 \%$ even at the shortest data acquisition window of $100 \mathrm{~ms}$. Imaging during mid-diastole may not be optimal for the RCA. This has been indicated by studies such as that from Herzog et al. [16].

\section{CONCLUSIONS}

The 4D NCAT phantom with its enhanced model for the cardiac motion provides a valuable tool to investigate the optimization of CT cardiac applications. Parameters such as the heart rate and motion and the location and size of cardiac plaques are all under user control thus providing a gold standard from which to evaluate and improve imaging techniques and methods. From our pilot simulation study using the phantom, we conclude that heart rate is an important determinant of image quality and artifact generation in MSCT imaging of the coronary arteries. It was found to contribute significantly to blurring and artifacts in MSCT images. Our results indicate the importance of

optimizing the data acquisition window with regard to heart rate and plaque location for improved CT images at a reduced patient dose.

\section{ACKNOWLEDGEMENTS}

This work was supported by grant number NIH R01 EB001838 from the National Institutes of Health (NIH). Its contents are solely the responsibility of the authors and do not necessarily represent the official views of the NIH.

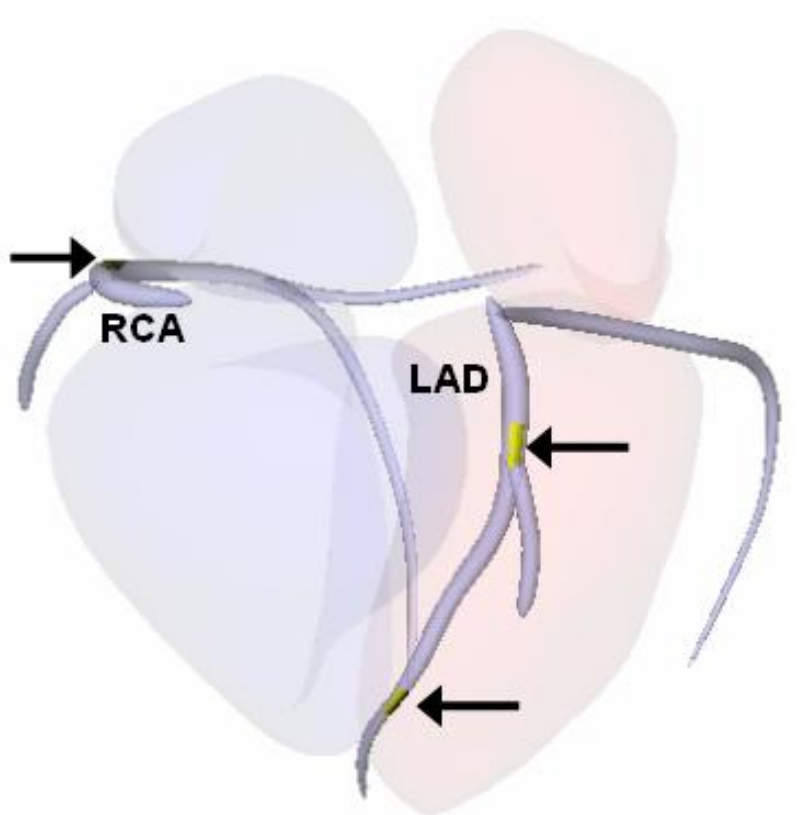

Figure 5: Locations of the plaques simulated in the 4D NCAT heart. A plaque was placed in the proximal RCA, the proximal LAD, and the distal LAD.

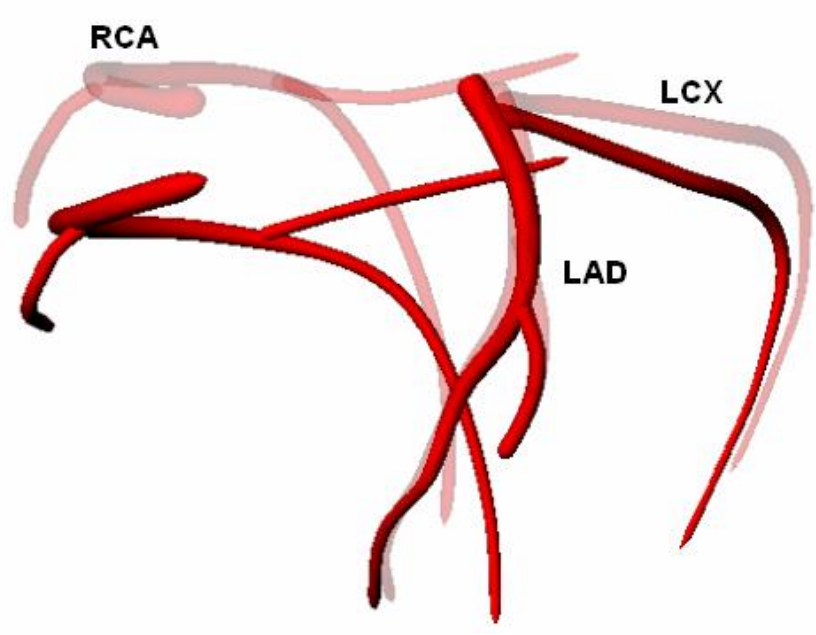

Figure 6: Motion of the coronary artery segments from end-diastole (faded red) to end-systole (red). 

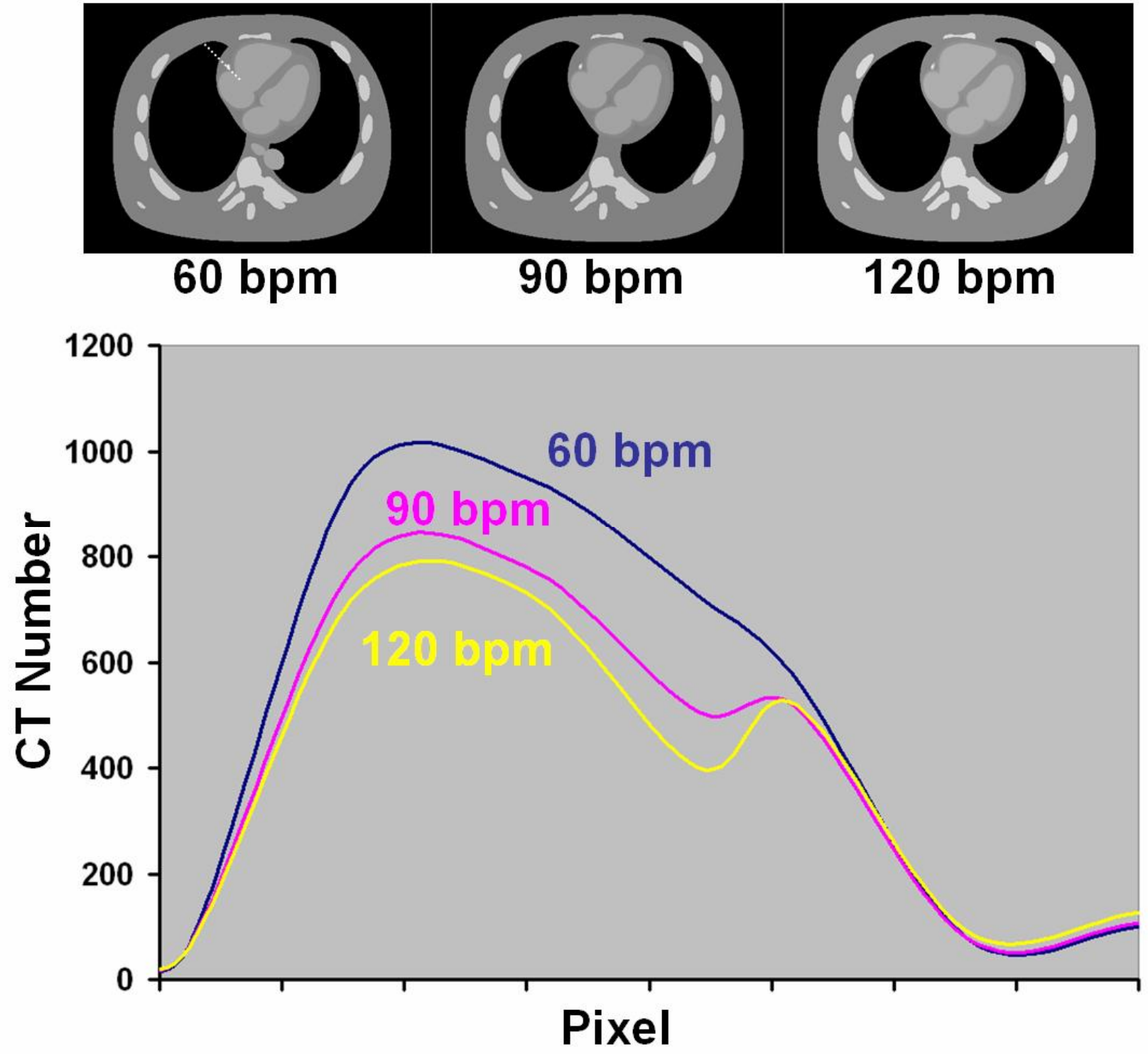

Figure 7: (Top) Noise-free CT simulations from the 4D NCAT modeling three different heart rates using a data acquisition window of $100 \mathrm{~ms}$. (Bottom) Profile (dotted line in the top figure) through the cardiac plaque modeled in the RCA. 


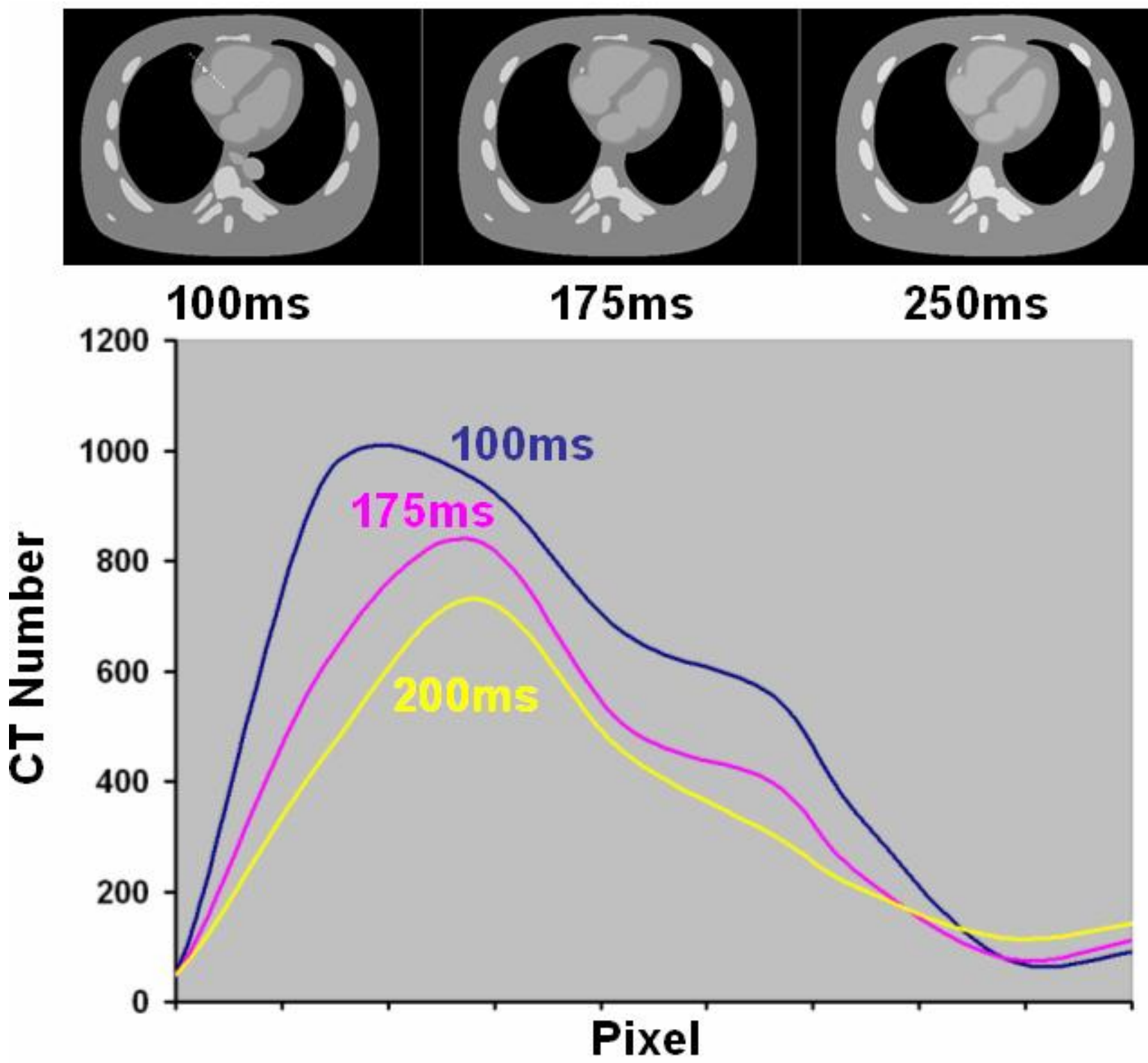

Figure 8: (Top) Noise-free CT simulations from the 4D NCAT modeling three different data acquisition windows with a heart rate of $60 \mathrm{bpm}$. (Bottom) Profile (dotted line in the top figure) through the cardiac plaque modeled in the RCA. 


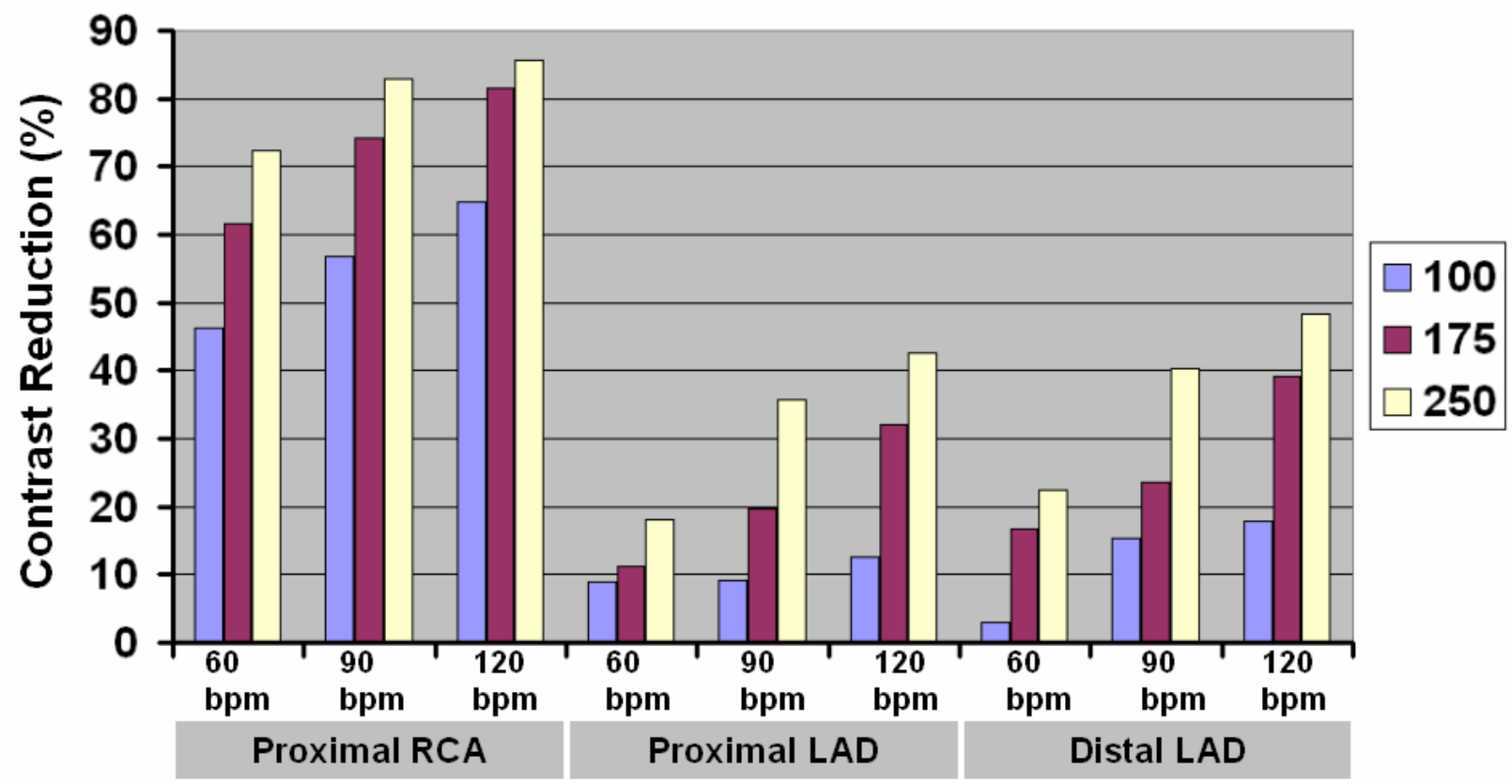

Figure 9: Reduction in plaque contrast measured from the different noise-free simulations using the three different heart rates $(60,90$, and $120 \mathrm{bpm})$ and data acquisition windows (100, 175, and 250ms).

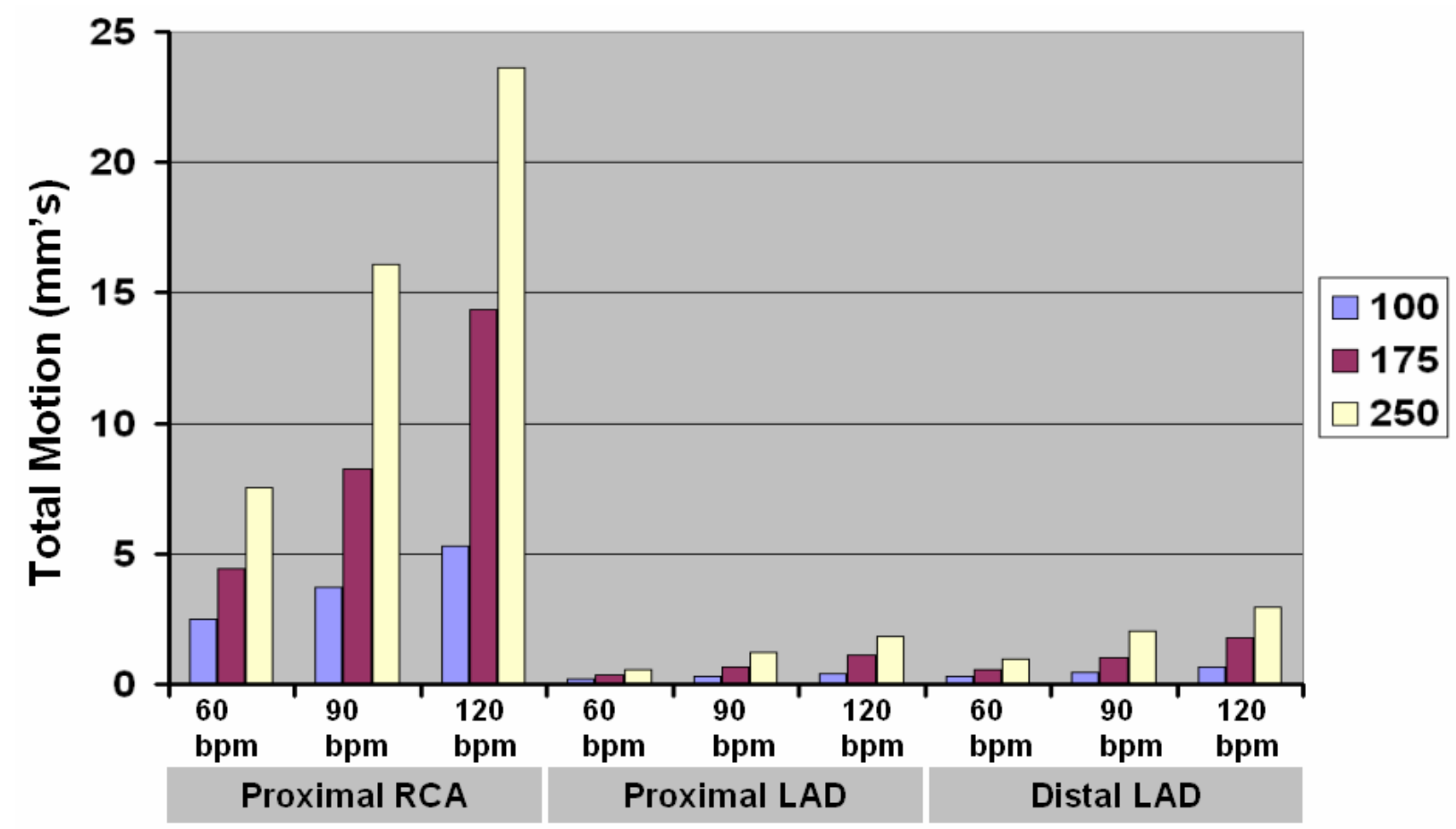

Figure 10: Total amount of motion of the cardiac plaques during the acquisition windows for each heart rate. The movement of the RCA is much more dramatic than that of the LAD. 


\section{REFERENCES}

1. Kalender, W., Computed Tomography. 2000, New York: Wiley \& Sons.

2. Ohnesorge, B., et al., Cardiac imaging with retrospectively ECG-gated fast multi-slice spiral CT. Radiology, 2000. 40(2): p. 111-117.

3. Schoepf, U.J., C.R. Becker, B.M. Ohnesorge, and E.K. Yucel, CT of Coronary Artery Disease. Radiology, 2004. 232: p.18-37.

4. Segars, W.P., D.S. Lalush, and B.M.W. Tsui, A realistic spline-based dynamic heart phantom. Ieee Transactions on Nuclear Science, 1999. 46(3): p. 503-506.

5. Segars, W.P., D.S. Lalush, and B.M.W. Tsui, Modeling respiratory mechanics in the MCAT and spline-based MCAT phantoms. Ieee Transactions on Nuclear Science, 2001. 48(1): p. 89-97.

6. Segars, W.P., Development and application of the new dynamic NURBS-based cardiac-torso (NCAT) phantom, in Biomedical Engineering. 2001, University of North Carolina: Chapel Hill, NC.

7. Moody, D. and S. Lozanoff, "SURFdriver: A practical computer program for generating three-dimensional models of anatomical structures," Paper presented at the $14^{\text {th }}$ Annual Meeting of the American Association of Clinical Anatomists, July 8-11, 1997.

8. McNeil, R. Rhinoceros: www.rhino3d.com, Seattle, WA, 1998.

9. Park, J., D.N. Metaxas, and L. Axel, Analysis of left ventricular wall motion based on volumetric deformable models and MRI-SPAMM. Medical Imaging Analysis, 1996. 1: p. 53-71.

10. Park, J., D.N. Metaxas, and L. Axel, Quantification and visualization of the 3D nonrigid motion of the left ventricle. in Proceedings of the SPIE Medical Imaging Conference (Physiology and Function), 1997: p. 177.

11. Park, J., et al., Deformable models with parameter functions for cardiac motion analysis from tagged MRI data. IEEE Transactions on Medical Imaging, 1996. 15: p. 278-289.

12. Young, A.A. and L. Axel, Three-dimensional motion and deformation of the heart wall: estimation with spatial modulation of magnetization--a model-based approach. Radiology, 1992. 185(1): p. 241-7.

13. Young, A.A., et al., Tracking and finite element analysis of stripe deformation in magnetic resonance tagging. IEEE Transactions on Medical Imaging, 1995. 14(3): p. 413-421.

14. Segars, W.P., M. Mahesh, T. Beck, E.C. Frey, and B.M.W. Tsui, "Validation of the 4D NCAT simulation tools for use in high-resolution X-ray CT research", in the Conference Record of the SPIE Medical Imaging Conference, San Diego, CA, 2005

15. Cranley, K., et al., Catalogue of Diagnostic X-ray Spectra and Other Data, Diagnostic Radiology and Magnetic Resonance Special Interest Group of the Institute of Physics and Engineering in Medicine.

16. Herzog, C., M. Arning-Erb, S. Zangos, K. Eichler, R. Hammerstingl, S. Dogan, H. Ackermann, and T. Vogl, "Multi-detector row CT coronary angiography: influence of reconstruction technique and heart rate on image quality", Radiology, vol. 238, pp. 75-86, 2006. 